\title{
Facoemulsificação em cães, com e sem implante de lente intra-ocular em piggyback: estudo clínico da inflamação pós-operatória ${ }^{1}$
}

\author{
Geórgia N. Rodrigues ${ }^{2}$, José Joaquim T. Ranzani ${ }^{3}$, Antonio Carlos L. \\ Rodrigues $^{4}$, Cláudia Valéria S. Brandão ${ }^{3}$, Daniela N. Cremonini ${ }^{2}$, Rosana \\ Maria O. Clark ${ }^{2}$ e Silvia Helena V. Perri ${ }^{5}$
}

\begin{abstract}
Rodrigues G.N., Ranzani J.J.T., Rodrigues A.C.L., Brandão C.V.S., Cremonini D.N., Clark R.M.O. \& Perri S.H.V. 2010. [Phacoemulsification in dogs with and without intraocular lens implantation in piggyback: Clinical study of postoperative inflammation.] Facoemulsificação em cães, com e sem implante de lente intra-ocular em piggyback: estudo clínico da inflamação pós-operatória. Pesquisa Veterinária Brasileira 30(2):103107. Departamento de Cirurgia e Anestesiologia Veterinárias, Faculdade de Medicina Veterinária e Zootecnia, Universidade Estadual Paulista, Campus de Botucatu, Botucatu, SP 18618-000, Brazil. E-mail: georgianr@uol.com.br

Perioperative and postoperative uveitis is the main problem in cataract surgery in dogs, affecting short-term and long-term postoperative success. Numerous therapeutic methods involving both steroidal and non-steroidal anti-inflammatory agents have been used to reduce surgically-induced uveitis. The purpose of this study was to investigate the postoperative inflammatory reaction and intraocular pressure after phacoemulsification surgery for cataract extraction with and without intraocular piggyback lens (IOL) implantation in dogs. A total of 25 dogs were divided into two groups: Group 1 (with IOL implantation) and Group 2 (without IOL implantation). The performed surgical technique consisted of unilateral bimanual phacoemulsification. Clinical assessment and intraocular pressure were measured before surgery (0) and at 3, 7, 14, 21, 28, 60 days after the surgery. Clinical assessment revealed inflammatory reaction more severe in dogs of $\mathrm{G} 1$ when compared to $\mathrm{G} 2$. Intraocular pressure did not differ significantly either between G1 and G2 or operated and non-operated eyes. Intraocular lens (IOL) implantation using piggyback technique with human IOL is a possible method to be performed in canine ophthalmology. However, nursing care is necessary as the the procedure induces inflammation and complications in the postoperative period.
\end{abstract}

INDEX TERMS: Dog, cataract, phacoemulsification, intraocular lens, intraocular pressure.

RESUMO.- A uveíte peri e pós-operatória é o maior problema da cirurgia para extração de catarata no cão, sendo considerada o fator mais importante para o sucesso cirúrgico,

\footnotetext{
${ }^{1}$ Recebido em 20 de maio de 2009.

Aceito para publicação em 18 de junho 2009

2 Faculdade de Medicina Veterinária e Zootecnia (FMVZ), Universidade Estadual Paulista (Unesp), Distr. Rubião Jr s/n, Botucatu, SP 18618-000, Brasil. *Autor para correspondência: georgianr@uol.com.br

${ }^{3}$ Departamento de Cirurgia e Anestesiologia Veterinárias, FMVZUnesp, Botucatu, SP 18618-000.

${ }^{4}$ Departamento de Oftalmologia, Otorrinolaringologia e Cirurgia de Cabeça e Pescoço, Faculdade de Medicina de Botucatu (FMB), Unesp, Botucatu, SP 18618-970.

5 Departamento de Apoio, Produção e Saúde Animal, Faculdade de Odontologia e Curso de Medicina Veterinária (FOA), Unesp, Araçatuba, SP 16050-680, Brasil.
}

imediato e tardio. Diversos protocolos pré e pós-operatórios utilizando agentes anti-inflamatórios esteroidais e não-esteroidais têm sido empregados na tentativa de controle da uveíte cirurgicamente induzida. O objetivo do presente estudo foi avaliar a reação inflamatória pós-operatória, clinicamente e por meio da pressão intraocular (PIO), após a cirurgia de facoemulsificação para extração de catarata em cães, com e sem implante de lente intraocular (LIO) em piggyback. Empregaram-se, 25 cães portadores de catarata, subdivididos em dois grupos: G1 (com implante de LIO), G2 (sem implante de LIO). A técnica cirúrgica adotada foi a facoemulsificação bimanual unilateral. Avaliações clínicas e mensurações da PIO foram aferidas antes do procedimento cirúrgico (0) e nos tempos 3, 7, 14, 21, 28 e 60 dias após o ato cirúrgico. Cães do grupo G1 apresentaram sinais clínicos 
de uveíte visivelmente mais intensos, relativamente aos do G2. Entretanto, a PIO não demonstrou diferença significativa entre os dois grupos analisados, nem entre os olhos operados e os contralaterais. A utilização de duas LIOs humanas em piggyback no cão é exequível, porém suscita mais inflamação e complicações no pós-operatório.

TERMOS DE INDEXAÇÃO: Cão, catarata, facoemulsificação, lente intraocular, pressão intraocular.

\section{INTRODUÇÃO}

A uveíte peri e pós-operatória é considerada o maior problema da cirurgia para extração de catarata no cão (Dziezyc 1990, Whitley et al. 1993, Williams et al. 1996, Gelatt \& Gelatt 2001, Moore et al. 2003). Ela é decorrente da interferência mecânica nos tecidos do segmento anterior do olho durante o ato cirúrgico e também da resposta imune ao material da lente que é liberado durante a cirurgia e que, muitas vezes, não é totalmente removido durante o procedimento. A penetração da câmara anterior e a subsequente perda de humor aquoso, com brusca redução da pressão intraocular (PIO), desencadeia a liberação de prostaglandinas pelo tecido uveal, o que resulta em miose e quebra da barreira hemato-aquosa com extravasamento, para o humor aquoso, de proteínas a partir dos vasos sanguíneos da íris e corpo ciliar (Williams et al. 1996, Gelatt \& Gelatt 2001).

O trauma físico decorrente da cirurgia intraocular determina a quebra da barreira hemato-aquosa e a liberação de mediadores químicos, tais como serotonina, histamina, plasmina, cininas e complemento. O ácido araquidônico liberado em decorrência do dano celular, vai produzir, através das vias cicloxigenase e lipoxigenase, mediadores inflamatórios, tais como prostaglandinas, tromboxano e leucotrienos. Certos mediadores químicos e inflamatórios determinam alterações na permeabilidade vascular, levando ao extravasamento de fluidos, proteínas e células a partir dos vasos sanguíneos da íris e corpo ciliar (Goodhead 1996, Williams et al. 1996, Gelatt 1999, Gelatt \& Gelatt 2001, Holzer et al. 2002). Sabe-se também que indivíduos portadores de catarata, principalmente aquelas maduras e hipermaduras, em início de reabsorção, possuem níveis mais elevados de anticorpos circulantes contra as proteínas da lente, comparativamente aos não portadores. Em face disso, esses indivíduos, após o ato cirúrgico, demonstram reações humorais e celulares bem mais exacerbadas (Williams et al. 1996).

A resposta imediata do olho ao trauma determinado pela cirurgia intraocular inclui quebra da barreira hematoocular, hiperemia ocular, miose e alterações na produção e drenagem do humor aquoso, traduzindo-se numa hipertensão ocular inicial seguida de prolongada hipotensão (Millichamp \& Dziezyc 1991).

A uveíte é o fator mais importante para o sucesso cirúrgico, pois afeta os resultados imediatos e tardios. Atualmente, as taxas de sucesso da cirurgia de facoemulsificação em cães situam-se em torno de $80 \%$ a $90 \%$, dimi- nuindo em cerca de $10 \%$ a $20 \%$ após três a cinco anos do ato cirúrgico (Gelatt \& Gelatt 2001). A facoemulsificação em cães portadores de uveíte induzida pela lente (uveítes facolíticas) apresenta uma redução nas taxas de sucesso para aproximadamente 52\% (Dziezyc 1990, Whitley et al. 1993, Van der Woerdt 2000, Gelatt \& Gelatt 2001, Leasure et al. 2001). Em decorrência disso, diversos protocolos pré e pós-operatórios, que utilizam agentes anti-inflamatórios esteroidais e não esteroidais, têm sido empregados na tentativa de controle da uveíte cirurgicamente induzida (Williams et al. 1996).

O diagnóstico das uveítes é basicamente clínico e leva em consideração sinais característicos ao exame oftálmico (Gelatt 1999, Leasure et al. 2001), como a turbidez do humor aquoso (flare), o efeito Tyndall, a dilatação retardada ou incompleta da pupila após instilação do colírio midriático (tropicamida 1\%) e a hipotensão ocular. Entretanto, a utilização científica de alguns desses parâmetros diagnósticos pode ser complicada, uma vez que tanto o flare como o efeito Tyndall, mesmo utilizando escalas de graduação são considerados métodos subjetivos, pois podem apresentar variação entre diferentes observadores. Além disso, a resposta pupilar ao colírio midriático não pode ser quantificada (Leasure et al. 2001). Procedimentos diagnósticos suplementares, como a flaremetria a laser e fluorfotometria, fornecem resultados quantitativos e precisos, no entanto, não são empregados rotineiramente devido à maior complexidade de execução, ao fato de serem invasivos, por requererem sedação profunda ou anestesia geral e por possuírem custo elevado (Leasure et al. 2001).

A redução da PIO é um achado clínico comum nas uveítes anteriores em cães. Estudos experimentais têm relacionado à diminuição da $\mathrm{PIO}$ com liberação de prostaglandinas e aumento da drenagem do humor aquoso, através da via úveo-escleral (Leasure et al. 2001). Em face disso, a tonometria tem sido considerada uma técnica muito útil, tanto para o diagnóstico como para o acompanhamento das uveítes em cães (Leasure et al. 2001). Alguns autores (Gelatt \& Gelatt 2001) preconizam a mensuração da PIO como uma das importantes avaliações na seleção de pacientes para cirurgia de facoemulsificação e consideram PIOs inferiores a $10-12 \mathrm{mmHg}$ como sinal de iridociclite. Nesses casos, terapia anti-inflamatória é instituída e a cirurgia indicada apenas quando a PIO apresentar valores entre $15-18 \mathrm{mmHg}$.

Este estudo objetivou avaliar a inflamação ocular pósoperatória do olho de cães frente à técnica de facoemulsificação com implantação de duas lentes intraoculares (LIOs) de silicone dobrável humanas em piggyback para correção da afacia. A escolha das LIOs humanas foi motivada pela ampla disponibilidade no mercado mundial e compatibilidade com as pequenas incisões utilizadas pela técnica de facoemulsificação.

\section{MATERIAL E MÉTODOS}

Integraram os grupos de estudo 25 cães portadores de catarata, machos e fêmeas, de várias raças e idades. Os cães foram 
subdivididos em dois grupos: Grupo 1 (G1) composto por 15 cães submetidos à cirurgia de facoemulsificação para extração da catarata, com implante de duas lentes intra-oculares de silicone dobráveis em piggyback; Grupo 2 (G2) composto por 10 cães submetidos à cirurgia de facoemulsificação sem implante de lentes intraoculares. Todos os cães foram submetidos a exames oftálmico e clínico geral, além de exames laboratoriais e avaliação cardíaca pré-operatória. Os cães que apresentavam doenças sistêmicas ou oculares que contraindicassem a cirurgia para extração da catarata foram excluídos deste estudo, bem como aqueles submetidos à cirurgia intraocular prévia e os diabéticos. A distribuição dos cães nos diferentes grupos foi aleatória. O protocolo realizado foi submetido e aprovado pela Câmara de Ética em Experimentação Animal (Protocolo $n^{\circ}$ 034/2000-CEEA).

O tratamento pré-operatório iniciou-se três dias antes da cirurgia e utilizou prednisona oral $(1 \mathrm{mg} / \mathrm{kg} / \mathrm{SID})$ e colírio de dexametasona e cloranfenicol (QID). No dia anterior ao ato cirúrgico iniciou-se antibioticoprofilaxia oral com enrofloxacina (5mg/kg/SID). A dilatação pupilar foi promovida com utilização de colírio de atropina $1 \%$, instilado uma vez na noite anterior e na manhã da cirurgia. Flunixin meglumine $(0,5 \mathrm{mg} / \mathrm{kg} / \mathrm{IV})$ foi aplicado 15 minutos antes do procedimento cirúrgico.

Todos os procedimentos cirúrgicos foram unilaterais e executados pelo mesmo cirurgião. Os cães foram submetidos à anestesia geral inalatória com isoflurano. A técnica cirúrgica adotada para remoção da catarata foi a de facoemulsificação bimanual, com duas incisões, uma principal tunelizada de 3,2mm e outra auxiliar, ambas localizadas em córnea clara. Empregaram-se durante a cirurgia Azul de Trypan para o auxílio da capsulorrexe, substâncias viscoelásticas (metilcelulose $2 \%$ e AV) para proteção dos tecidos intraoculares, manutenção dos espaços e auxílio no implante das LIOs e solução salina balanceada para irrigação intraocular. No Grupo 1 procedeu-se o implante de duas LIOs de silicone dobrável ${ }^{6}$, em piggyback, através da incisão principal, com utilização de injetor ${ }^{7}$. As zonas ópticas foram posicionadas no interior do saco capsular, capturadas na capsulorrexe anterior, mantendo as alças no sulco ciliar. A sutura da incisão principal da córnea foi executada com dois pontos simples separados e sepultados, utilizando-se fio mononáilon 10-0.

O protocolo pós-operatório foi constituído de prednisona oral, iniciando-se na dose de $1 \mathrm{mg} / \mathrm{kg} / \mathrm{BID}$, com redução gradativa e retirada aos 28 dias de pós-operatório, enrofloxacina oral, na dose de $5 \mathrm{mg} / \mathrm{kg} / \mathrm{SID}$ por 10 dias, colírio de atropina $1 \%$, diariamente durante sete dias, e colírio de dexametasona e cloranfenicol, inicialmente a cada duas horas, seguido de reduções graduais. Analgesia utilizando cloridrato de tramadol $(2 \mathrm{mg} / \mathrm{kg})$ também foi procedida.

Os cães foram submetidos a avaliações oculares por meio de exame oftálmico padrão antes do procedimento cirúrgico (momento 0) e após 3, 7, 14, 21, 28 e 60 dias do ato cirúrgico. A mensuração da PIO, expressada em $\mathrm{mmHg}$, foi procedida na área central da córnea de ambos os olhos empregando-se o tonômetro de aplanação ${ }^{8}$ e seu valor representou a média de três aferições consecutivas, com desvio padrão de $5 \%$. As mensurações foram feitas antes do procedimento cirúrgico,

\footnotetext{
${ }^{6}$ AMO PhacoFlex II, SI 40 NB, AMO Advanced Medical Optics, São Paulo, SP, Brasil.

7 The Unfolder Silver Series, Allergan, Irvine, CA, USA.

8 Tonopen XL, Mentor Inc, Norwell, Mass, USA.
}

estabelecendo-se tal medida como momento zero (0). As demais aferições foram procedidas aos 3, 7, 14, 21, 28 e 60 dias após ato cirúrgico. Os dados referentes às mensurações da PIO foram submetidos à análise de variância com medidas repetidas, sendo as médias comparadas pelo teste de Tukey com nível de significância de 5\% (ZAR 1998). As análises estatísticas foram efetuadas empregando-se o programa SAS (Statistical Analysis System).

\section{RESULTADOS}

\section{Avaliação clínica}

Todos os olhos operados, do G1 e G2, apresentaram sinais de uveíte no pós-operatório, porém, visivelmente mais exacerbados nos cães do G1. Os sinais clínicos incluíram hiperemia conjuntival, edema corneano, flare, presença de fibrina na câmara anterior, formação de membranas fibropupilares, sinéquias posteriores de íris, íris bombé, irregularidade pupilar e opacidade vítrea, além de sinais de desconforto ocular, tais como, lacrimejamento, blefarospasmo e fotofobia. Relativamente à presença de fibrina na câmara anterior, irregularidade pupilar e sinéquias posteriores de íris, $75 \%$ dos casos ocorreram em olhos de cães do G1.

De maneira geral, os sinais clínicos manifestaram-se mais severos nos primeiros dias do pós-operatório, tendendo à redução gradativa ao longo do tempo; no entanto, a quase totalidade dos olhos operados ainda apresentava algum sinal de uveíte ao final do período de observação (60 dias de pós-operatório). Outras complicações pósoperatórias, relacionadas ou não à uveíte, também ocorreram, tais como bloqueio pupilar, glaucoma, descolamento de retina, úlcera corneana e opacidade das cápsulas anterior (borda da capsulorrexe) e posterior da lente.

\section{Pressão intraocular}

A PIO dos olhos operados não demonstrou diferença significativa entre os grupos em nenhum dos momentos avaliados; no entanto, nos cães do G2 observou-se diferença significativa entre os momentos 0 e 60 , revelando uma redução da $\mathrm{PIO}$ aos 60 dias de pós-operatório em comparação com os valores prévios ao procedimento cirúrgico (Fig.1).

Comparações da PIO entre olhos operados e contralaterais (não operados) não revelaram diferenças em ne-

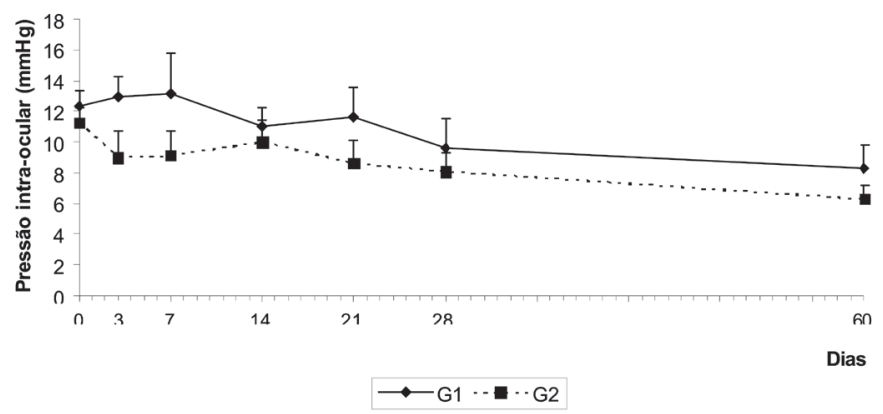

Fig.1. Pressão intraocular média $(\mathrm{mmHg})$ dos olhos operados, Grupos 1 e 2, segundo momentos de observação (dias de pós-operatório). 


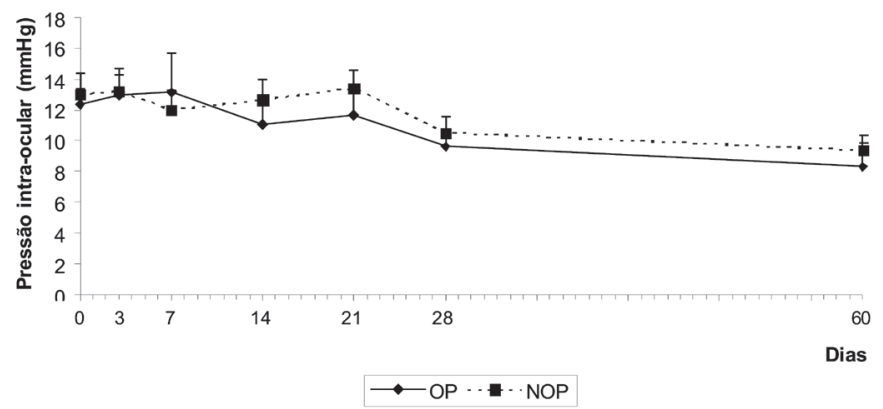

Fig.2. Pressão intraocular média $(\mathrm{mmHg})$ dos olhos operados e contralaterais (não operados) dos animais do Grupo 1, segundo momentos de observação (dias de pós-operatório).

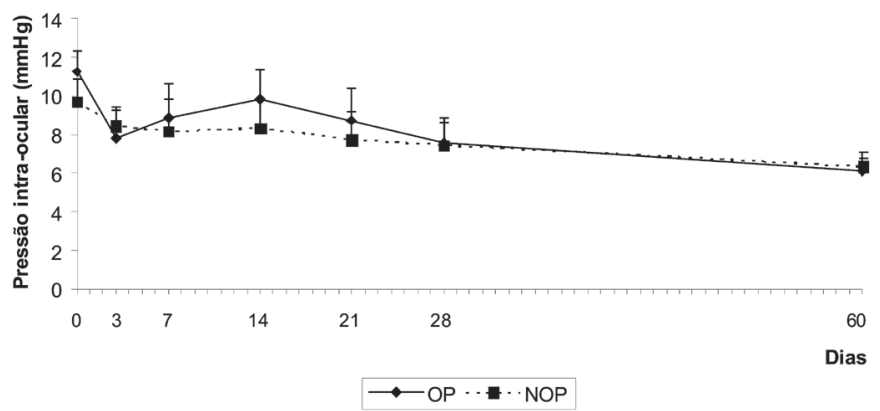

Fig.3. Pressão intraocular média $(\mathrm{mmHg})$ dos olhos operados e contralaterais (não operados) dos animais do Grupo 2, segundo momentos de observação (dias de pós-operatório).

nhum dos momentos avaliados, tanto nos cães do G1 (Fig.2) como nos do G2 (Fig.3). Entretanto, nos cães de G1 observaram-se diferenças de PIO entre os momentos 0 e 60 dos olhos não operados (Fig.2).

\section{DISCUSSÃO}

As técnicas cirúrgicas utilizadas para remoção da catarata têm sido modificadas com o passar dos anos, sempre no intuito de aumentar as taxas de sucesso do procedimento, com diminuição de suas complicações. Atualmente, a facoemulsificação é a técnica de escolha para remoção da catarata que, utilizando pequenas incisões em córnea clara, reduz o trauma físico e, consequentemente, a inflamação pós-operatória, o que melhora os resultados pós-operatórios (Bistner 1992, Williams et al. 1996, Glover \& Constantinescu 1997, Holzer et al. 2002).

A elevação nos índices de sucesso da cirurgia para extração da catarata impulsionou a busca de melhores resultados referentes à acuidade visual, o que determinou a utilização de lentes intraoculares (LIOs) (Whitley et al. 1993, Glover \& Constantinescu 1997, Davidson 2001). Acompanhando a tendência de incisões menores, de 2,5 a $3,5 \mathrm{~mm}$, que induzem menor astigmatismo, surgiram as LIOs dobráveis, primeiramente as de silicone e, seguidamente, as de diferentes polímeros de acrílico (Davidson 2001). A grande dificuldade no desenvolvimento de lentes dobráveis para cães reside no alto poder dióptrico da
LIO necessário para a espécie, em torno de $40 \mathrm{D}$, o que torna difícil sua compatibilidade com as pequenas incisões, empregadas na facoemulsificação (Davidson 2001).

A implantação de duas ou mais LIOs de câmara posterior, denominada polipseudofacia ou piggyback, foi introduzida na cirurgia humana por Gayton \& Sanders em 1993. O objetivo das lentes em piggyback era promover correção pseudofácica adequada em pacientes que necessitavam de lentes de alto poder dióptrico e possibilitar a correção secundária em casos de resultado óptico insatisfatório (Gills \& Fenzl 1999, Gayton et al. 2000, Donoso \& Rodrígues 2001). O procedimento de piggyback também tem sido considerado vantajoso, do ponto de vista óptico, comparativamente à implantação de uma única LIO com alto poder dióptrico, uma vez que no homem é conhecido o fenômeno de aberrações ópticas causadas por implantação de LIO única com alto poder dióptrico (Donoso \& Rodrígues 2001).

A técnica de piggyback para implante de LIOs em cirurgia de facoemulsificação em cães poderia revelar-se uma solução procedente para correção da afacia, uma vez que viabilizaria a utilização LIOs dobráveis humanas, amplamente disponíveis no mercado, compatíveis com pequenas incisões e, ao mesmo tempo, capazes de atingir o alto poder dióptrico necessário para a espécie. As pequenas incisões empregadas na técnica de facoemulsificação são fundamentais no que se refere à redução do trauma cirúrgico e, consequentemente, da uveíte pós-operatória.

Apesar da pressão intra-ocular avaliada neste estudo não ter demonstrado diferença significativa nos olhos operados quando comparados os períodos pré e pós-operatório, nem diferença entre os grupos avaliados, o comportamento clínico dos olhos durante o pós-operatório diferiu bastante entre os grupos. Os cães do Grupo 1 demonstraram pós-operatório mais complicado, apresentando reação inflamatória mais exacerbada e prolongada, relativamente aos que não receberam o implante das LIOs. As complicações pós-operatórias também foram mais frequentes nos cães que receberam as LIOs, fato este, muito provavelmente, decorrente da maior inflamação manifestada após o ato cirúrgico.

As causas da disparidade da intensidade da inflamação pós-operatória entre os grupos, no entanto, não puderam ser determinadas, porém algumas suspeitas surgiram. O tempo cirúrgico mais prolongado e a maior manipulação dos tecidos intraoculares a que foram submetidos os cães do G1, muito provavelmente influenciaram a uveíte pós-operatória e, consequentemente, os resultados cirúrgicos. A implantação de duas LIOs em piggyback naturalmente requer maior manipulação; a utilização de LIOs humanas em cães intensificou o problema, pois devido à diferença de tamanho (LIOs específicas para cães são maiores) foi necessário "capturar" a zona óptica das LIOs na capsulorrexe, para evitar sua descentração, o que acarretou em mais manipulação intraocular. Segundo alguns autores (Gelatt \& Gelatt 2001), quanto maior a manipulação e o trauma cirúrgico na íris durante o ato cirúr- 
gico, maior é a iridociclite resultante no pós-operatório e, consequentemente, menor é o sucesso cirúrgico. As LIOs caninas, devido ao seu elevado poder dióptrico (aproximadamente +41D), exigem zonas ópticas maiores comparativamente às LIOs humanas, isto para que tolerem leves descentrações sem causarem aberrações ópticas (Gelatt \& Gelatt 2001).

Outro fator que supostamente poderia ter influenciado a inflamação pós-operatória, foi a quantidade de substância viscoelástica utilizada, uma vez que a maior manipulação para implantação das LIOs nos cães do G1 exigiu uma utilização bem maior de viscoelástico, em relação aos do G2. Além disso, a própria LIO implantada pode ter desempenhado algum papel no comportamento dos olhos do G1 no pós-operatório, pois, segundo alguns autores (Gelatt \& Gelatt 2001), a composição do material da LIO pode influenciar o desenvolvimento de fibrose capsular em cães no pós-operatório, entretanto, a literatura é escassa de estudos clínicos neste sentido.

A catarata hipermatura é o tipo de catarata mais frequentemente extraída no cão. Infelizmente, a liberação de material da lente nessa fase resulta em uma variável e, muitas vezes, intensa uveíte faco induzida antes e após a cirurgia (Gelatt \& Gelatt 2001).

\section{CONCLUSÕES}

Conclui-se que o procedimento de implante de duas lentes intraoculares de silicone dobrável humanas em piggyback é possível de ser executado para correção da afacia após a extração da catarata em cães. No entanto, o procedimento requer habilidade na confecção da capsulorrexe da cápsula anterior, a fim de que a mesma possua tamanho correto para manter as zonas ópticas das LIOs em posição adequada, ou seja, fixas dentro do saco capsular. A "captura" das duas LIOs na capsulorrexe, fator imprescindível para o posicionamento adequado das LIOs em relação à pupila e eixo visual, demonstrou significativa dificuldade técnica.

A técnica de facoemulsificação com implante de duas LIOs em piggyback determina um tempo cirúrgico superior em relação ao não implante de LIO, o que culmina em uma maior manipulação intraocular.

A PIO dos olhos operados não se altera significativamente quando comparados os momentos anterior e posterior à cirurgia, o mesmo ocorrendo na comparação entre os olhos operados e seus contralaterais nos diferentes momentos avaliados.

Os sinais clínicos, oriundos de reação inflamatória ocular, as consequências e as complicações cirúrgicas foram mais acentuados nos olhos em que foram implantadas as lentes intraoculares em piggyback.
Agradecimentos.- À Fundação de Amparo à Pesquisa do Estado de São Paulo (FAPESP) pela concessão do Auxílio à Pesquisa (Proc. $\mathrm{n}^{\circ}$ 00/15079-1) e da bolsa de doutorado (Proc. $n^{\circ}$ 00/07449-3).

\section{REFERÊNCIAS}

Bistner S.I. 1992. Recent developments in comparative ophthalmology. Compend. Cont. Educ. Pract. Vet. 14(10):1304-1323.

Davidson M.G. 2001. Towards a better canine intraocular lens. Vet. Ophthalmol. 4(1):1.

Donoso R. \& Rodrígues A. 2001. Piggyback implantation using the AMO Array multifocal intraocular lens. J. Cataract Refract. Surg. 27(9):15061510.

Dziezyc J. 1990. Cataract surgery. Current approaches. Vet. Clin. North Am., Small Anim. Pract. 20(3):737-754.

Gayton J.L. \& Sanders V.N. 1993. Implanting two posterior chamber intraocular lenses in a case of microphthalmos. J. Cataract Refract. Surg. 19:776-777.

Gayton J.L., Apple D.J., Peng Q., Visessook N., Sanders V., Werner L., Pandey S.K., Escobar-Gomez M., Hoddinott D.S.M. \& Van Der Karr M. 2000. Interlenticular opacification: Clinicopathological correlation of a complication of posterior chamber piggyback intraocular lenses. J. Cataract Refract. Surg. 26(3):330-336.

Gelatt K.N. 1999. Veterinary Ophthalmology. $3^{\text {rd }}$ ed. Lippincott, Williams and Wilkins, Philadelphia, p.755-795.

Gelatt K.N. \& Gelatt J.P. 2001. Small Animal Ophthalmic Surgery: Practical techniques for the veterinarian. Butterworth-Heinemann, Woburn, p.286-334.

Gills J.P. \& Fenzl R.E. 1999. Minus-power intraocular lenses to correct refractive errors in myopic pseudophakia. J. Cataract Refract. Surg. 25(9):1205-1208.

Glover T.D. \& Constantinescu G.M. 1997. Surgery for cataract. Vet. Clin. North Am., Small Anim. Pract. 27(5):1143-1173.

Goodhead A.D. 1996. Uveitis in dogs and cats: Guidelines for the practitioner. J. S. Afr. Vet. Assoc. 67(1):12-19.

Holzer M.P., Solomon K.D., Sandoval H.P. \& Vroman D.T. 2002. J. Cataract Refract. Surg. 28(1):93-99.

Leasure J., Gelatt K.N. \& MacKay E.O. 2001. The relationship of cataract maturity to intraocular pressure in dogs. Vet. Ophthalmol. 4(4):273-276.

Millichamp N.J. \& Dziezyc J. 1991. Mediators of ocular inflammation. Progr. Vet. Comp. Ophthalmol. 1(1):41-58.

Moore D.L., McLellan G.J. \& Dubielzig R.R. 2003. A study of the morphology of canine eyes enucleated or eviscerated due to complications following phacoemulsification. Vet. Ophthalmol. 6(3):219226.

Whitley R.D., McLaughlin S.A., Whitley E.M. \& Gilger B.C. 1993. Cataract removal in dogs: The surgical techniques. Vet. Med. 88(9):859866.

Williams D.L., Boydell I.P. \& Long R.D. 1996. Current concepts in the management of canine cataract: A survey of techniques used by surgeons in Britain, Europe and the USA and a review of recent literature. Vet. Rec. 138(15):347-353.

Van der Woerdt A. 2000. Lens-induced uveitis. Vet. Ophthalmol. 3(4):227234.

Zar J.H. 1999. Biostatistical Analysis. $4^{\text {th }}$ ed. Prentice-Hall, New Jersey, p.210-260. 